\title{
Fatigue crack deflection in cruciform specimens subjected to biaxial loading conditions
}

\author{
Eric BREITBARTH*, Michael BESEL ${ }^{1}$ \\ German Aerospace Center (DLR), Institute of Materials Research, Linder Hoehe, 51147 Cologne, Germany \\ *eric.breitbarth@dlr.de,+49 (0)2203-601-2504 \\ michael.besel@dlr.de,+49 (0)2203-601-2068
}

Highlights:

- Biaxial testing of cruciform specimen to study crack deflection

- Analyzation of $T$-stress with finite element simulations

- Testing of fatigue cracks in the aluminum alloys AA2024-T351 and AA5028-H116

- Prediction of crack paths by simulations

Crack deflection is studied for the aluminum alloys AA2024-T351 and AA5028-H116 under biaxial fatigue loading. With cruciform specimens that have a testing area of about $420 \times 420 \mathrm{~mm}^{2}$ a fatigue crack was subjected to nominal biaxiality ratios between 1.0 and 3.0. Consequently, the crack is subjected to different magnitudes of T-stress. Finite element simulations of the growing crack revealed that the crack path is not significantly influenced by the T-stress but the T-stress predicts whether the crack path remains stable. If at least two crack paths are basically possible the crack will kink for positive T-stresses and remain stable for negative T-stresses. Furthermore, small spikes with strain concentrations along the crack path are identified with digital image correlation which might cause crack deflection in this case.

Keywords: Biaxial testing, Fatigue crack, Crack deflection, Aluminum, T-stress

\section{Introduction}

For commercial aircrafts the damage tolerance design and design against fatigue are necessary for safety reasons but they cause also economic problems in terms of cost-efficiency [1]. Such lightweight structures are subjected to loadings close to their limits for an optimal exploitation of their mechanical capabilities. Due to non-constant service loads during the service life fatigue cracks will arise and need to be considered in the design process. Consequently, not only the crack propagation rate but also the crack path needs to be predictable. In this context crack turning has been identified as an approach to enhance the damage tolerance behavior [2]. Dependent on the external stress state a turning crack can significantly enhanced the residual strength of fuselage structures with a 2-bay-crack, as the crack tip loadings are reduced [3], [4].

Under linear-elastic conditions the stress state near the crack tip can be analytically described with the Williams series expansion [5]. In linear-elastic fracture mechanics usually only the first term is used because the fracture behavior is dominated by the near-tip stress field. The coefficients are the wellknown stress intensity factors $K_{\mathrm{I}}$ and $K_{\mathrm{II}}$ with the $1 / \sqrt{\mathrm{r}}_{\mathrm{r}}$ singularity [6]. Taking into account the following non-singular coefficient leads to a stress component acting parallel to the crack growth direction. This constant stress term is known as the $T$-stress. Figure 1 illustrates the local stress components at the crack tip which lead to $K_{\mathrm{I}}, K_{\mathrm{II}}$ and the $T$-stress. It has to be mentioned that there is no equivalent term in the perpendicular direction because the related term in the Williams series is zero.

\footnotetext{
${ }^{1}$ Present affiliation: Otto Fuchs KG, Germany
} 


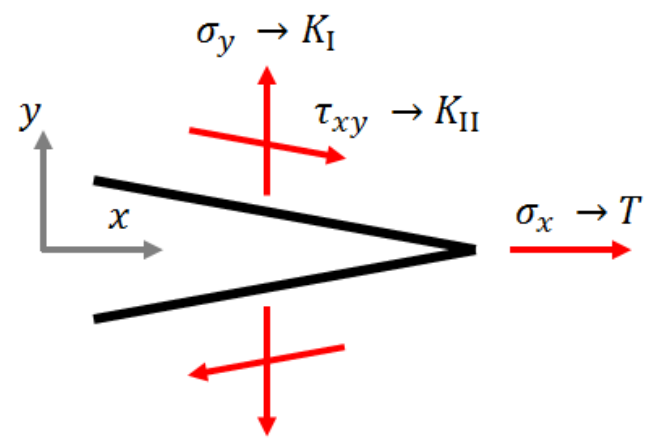

Figure 1: Illustration of stress components at the crack causing $K_{\mathrm{I}}, K_{\mathrm{II}}$ and $T$

Over time, it was found out that more accurate approaches including higher order terms are required to sufficiently capture local effects on the crack tip [7]. There are concepts that use up to three parameters in linear-elastic as well as in elastic-plastic fracture mechanics [8]. The $T$-stress has been identified as a parameter that can be used to predict the crack path stability under biaxial loadings. Under negative $T$-stress $(T<0)$ the crack path will remain stable while under positive $T$-stress $(T>0)$ the initial crack path becomes unstable [7], [9]. In linear-elastic fracture mechanics it is assumed that a crack grows straight under pure mode I loading conditions, i.e. in absence of mode II and III loadings. One criteria to predict the crack path taking into account the $T$-stress uses the maximum average tangential stress (MATS)). Here it is assumed that the crack will grow perpendicular to the direction where the tangential stress is maximum [10], [11]. Considering a straight crack orientated to one of the principal axis in a biaxially loaded sheet (like the skin of the fuselage structure of an aircraft), an evaluation of this loading situation only based on the concept of stress intensity factors will not account for any crack deviation effects, i.e. the crack will not change its direction within this kind of analysis. But taking into account the $T$-stress would allow the prediction of crack kinking starting at a certain stress level. Furthermore, the effects of $T$-stress are also clearly reflected in the shape of the plastic zone at the crack tip. While positive $T$-stress reduces the size of the plastic zone, negative $T$ stress increases its size [12]. The characteristics of the plastic zone again are very important as they significantly influence the crack behavior and can also be used to predict the crack path [13], [14]. In this paper the crack deflection under biaxial fatigue loading conditions is studied. Numerical analyses as well as local DIC investigations are carried out to investigate the crack propagation behavior of the aluminum alloys AA2024-T351 and AA5028-H116. Both alloys are used in the aviation industry, and here biaxial loading significantly influences the damage tolerance capability of the aircraft structures.

\section{Experimental Program \& Numerical Studies}

In the experimental program crack deflection was studied for the aluminum alloys AA2024-T351 and AA5028-H116. While the former had a thickness of $t=1.8 \mathrm{~mm}$ the latter had a thickness of $t=3.3 \mathrm{~mm}$. The experiments were supported by crack propagation simulations within a linear-elastic finite element model. The design of the specimen is shown in Figure 2. This kind of specimen has been used for decades at the German Aerospace Center with different modifications [15]. The gauge area of the specimen is in its center (blue part). With the dimension of $380 \mathrm{~mm}$ x $380 \mathrm{~mm}$ it covers the area of about three stringers as found in a fuselage structure. This (blue) sheet is joined to in total eight loading arms (grey - four on the front and four on the rear) that are fixed to the clamping tools of the testing machine. Finally, the cruciform specimen has overall dimensions of $1120 \mathrm{~mm}$ x $1120 \mathrm{~mm}$. The fatigue crack will grow inside this testing area in the center (blue) as illustrated by the red line in Figure 2. To promote the initial crack growth direction being parallel to one of the principal loading axis a starter notch was induced. Therefore, an $8 \mathrm{~mm}$ hole was drilled in the center of the specimen and $10 \mathrm{~mm}$ long saw cuts were introduced in the $\mathrm{x}$ direction on two opposite sides of this hole. 
The biaxial stress conditions are characterized by Equation 1 using the biaxiality ratio $\lambda$. It is defined by the stress parallel to the crack $\sigma_{=}$divided by the stress perpendicular to the crack $\sigma_{\perp}$.

$$
\lambda=\frac{\sigma_{=}}{\sigma_{\perp}}=\frac{\sigma_{\mathrm{x}}}{\sigma_{\mathrm{y}}}
$$

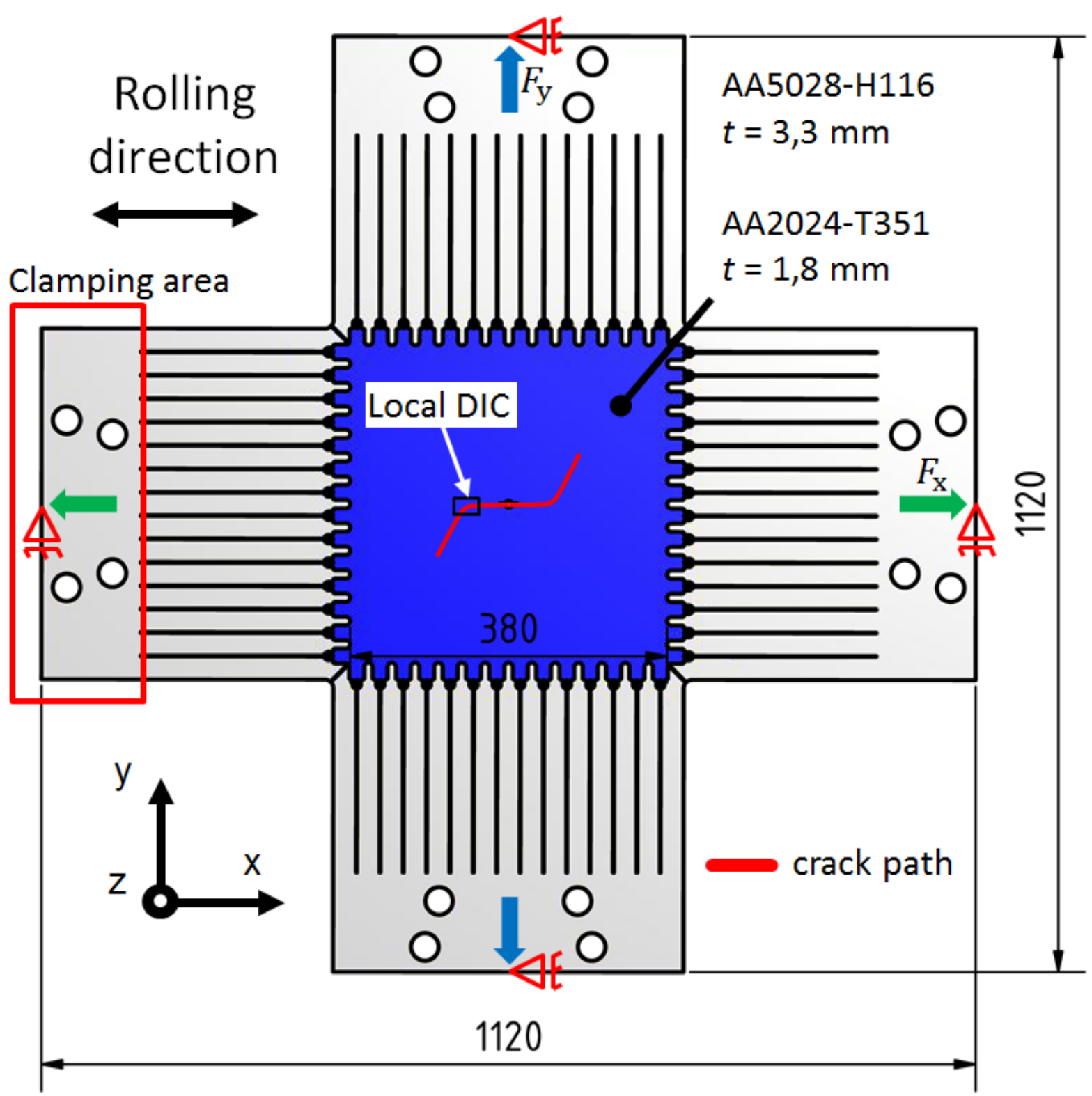

Figure 2: Details of the biaxial specimens with boundary conditions. The crack path of the fatigue crack experiment is illustrated in red.

In detail the following investigations were conducted in this paper:

(1) Finite element simulations of different angels of a kinking crack to analyze the relationship of $K_{\mathrm{I}}, K_{\mathrm{II}}$ and T-stress

(2) Crack propagation simulations in cruciform specimens with different biaxiality ratios $\lambda$ ranging from 1.0 to 3.0 . 
(3) Experimental investigations of the biaxiality ratios $\lambda$ ranging from 1.0 to 3.0 in specimens made of the aluminum alloys AA2024-T351 $(t=1.8 \mathrm{~mm})$ and AA5028-H116 $(t=3.3 \mathrm{~mm})$ under fatigue loadings with a load ratio $R$ of 0.1 .

a. Introducing a $2 a=80 \mathrm{~mm}$ long fatigue crack with $\Delta K_{\mathrm{I}}=10 \mathrm{MPa} \sqrt{\mathrm{m}}$ and $\lambda=0.0$

b. Crack propagation of $\Delta a=2-3 \mathrm{~mm}$ with $\lambda=1.0$

c. Crack propagation of $\Delta a=2-3 \mathrm{~mm}$ with $\lambda=1.5$ (only AA5028-H116 specimen)

d. Crack propagation of $\Delta a=2-3 \mathrm{~mm}$ with $\lambda=2.0$

e. Crack propagation of $\Delta a=2-3 \mathrm{~mm}$ with $\lambda=3.0$

f. Continuing with crack propagation with last biaxiality ratio after the crack kinked

With regards to aircraft structures the case of $\lambda=1.0$ can basically be attributed to parts of the upper fuselage section where hoop stresses and longitudinal stresses are nearly equal. A biaxiality ratio of $\lambda=2.0$ is a typical case for a circumferential crack if the stresses are estimated by Barlow's formula for a closed (thin walled) pipe-like structure with internal pressure. The stresses in hoop direction are twice as much as that in longitudinal direction. Complex load spectra representing realistic flight scenarios are neglected in these fundamental analyses as their consideration would clearly complicate the interpretation of the experimental findings. The experiments were conducted with two different initial stress states for both specimens which are $\sigma_{\mathrm{y}}=40 \mathrm{MPa}$ for the AA2024-T351 and $\sigma_{\mathrm{y}}=29 \mathrm{MPa}$ the AA5028-H116 specimen. For both experiments the corresponding stresses are finally summarized in Figure 8.

\section{$\underline{\text { Finite Element Simulations }}$}

All simulations were conducted with ANSYS Classic controlled by APDL scripts. The FE model of the cruciform specimen is derived from the geometry shown in Figure 2. Here the gauge area in the middle (blue section) is modelled with 2D plane stress elements. With thicknesses of $1.8 \mathrm{~mm}$ and $3.3 \mathrm{~mm}$ this sheet is thin compared to its overall dimensions (Figure 2) which supports this assumption of prevailing plane stress conditions. Because of the symmetry of the specimen out-of-plane effects like bending or buckling are limited to the very final stage of the experiments, and are consequently neglected in the simulations. Furthermore, $2 \mathrm{~d}$ elements have the advantage in fracture mechanical analyzes that only a unique value for the stress intensity factor is obtained instead of a through thickness distribution along the crack front for a $3 \mathrm{~d}$ model (which would require additional assumptions for the computation of a single representative stress intensity factor). The loading arms are modelled with $3 \mathrm{~d}$ solid elements because this element type facilitates the model generation as the loading arms can directly be coupled to both sides (i.e. upper and lower surface) of the testing area. Figure 2 also illustrates the boundary conditions of the model. The machine forces were applied on all loading arms in the clamping area (red rectangle). Furthermore, the displacements of the nodes were coupled in the clamping areas to represent a rigid clamping situation, and the displacement components perpendicular to the corresponding loading direction were suppressed. This should suitably represent the clamping conditions in the testing machine, please see Figure 3 for details. All simulations were conducted using a linear-elastic material model with a Young's modulus $E$ of $71 \mathrm{GPa}$ and a Poisson's ratio $v$ of 0.33 [16]. The geometry of the crack path was controlled with an external data file. Its content (i.e. the crack path) was controlled by a Python script. Repeated execution of this procedure simulated the incremental crack propagation under fatigue loading. After each simulation the crack tip loadings in terms of $K_{\mathrm{I}}$ and $K_{\mathrm{II}}$ were exported. Then the new crack propagation direction was calculated by Equation 2 [17] without any additional restrictions concerning the crack path's shape or curvature. With a step size of $2 \mathrm{~mm}$ the new crack path was calculated. In the next simulation loop the new crack path is read in again. The step size should be small compared to the overall length of the crack path. Here the increment of $2 \mathrm{~mm}$ corresponds to $1 \%$ of the maximum length of a straight crack in the specimen $(a \approx 200 \mathrm{~mm})$. 


$$
\theta_{c}=\mp\left[155,5^{\circ} \cdot \frac{\left|K_{\mathrm{II}}\right|}{\left|K_{\mathrm{I}}\right|+\left|K_{\mathrm{II}}\right|}-83,4^{\circ} \cdot\left(\frac{\left|K_{\mathrm{II}}\right|}{\left|K_{\mathrm{I}}\right|+\left|K_{\mathrm{II}}\right|}\right)^{2}\right]
$$

\section{$\underline{\text { Biaxial Fatigue Tests }}$}

The experimental setup is shown in Figure 3. The biaxial testing machine from Carl Schenck AG has four hydraulic cylinders which are crosswise arranged. It has a maximum loading capacity of $1000 \mathrm{kN}$ and $630 \mathrm{kN}$ on the main (x-direction) and secondary axis (y-direction), respectively. Together with the digital Instron 8800 controller even under biaxial loadings the specimen (1) is kept in the center. During the fatigue test the specimen was loaded in phase with a frequency of $5 \mathrm{~Hz}$. A GOM Aramis [18] digital image correlation system was used to compute the local deformation field around the crack path and even the plastic zone. For this purpose a Zeiss STEMI SV8 light microscope (2) together with a Canon EOS 1000D was used to take images of the local stochastic b/w pattern applied to the gauge area. The reference images for the DIC analysis were taken from the initially pristine material, i.e. without a crack, and the DIC-computed strains therefore represent total strain. The microscope (2) is mounted on a manual positioning system (3) that is used to follow the crack during the experiment. To observe the whole deformation field of the gauge area a GOM ARAMIS 12M 3d stereo correlation system was positioned above the specimen (not visible in Figure 3, but the interested reader is refered to [15] for further details). During the test the microscope shown in Figure 3 was removed in regular intervals to conduct full field measurements with the $12 \mathrm{M}$ system.

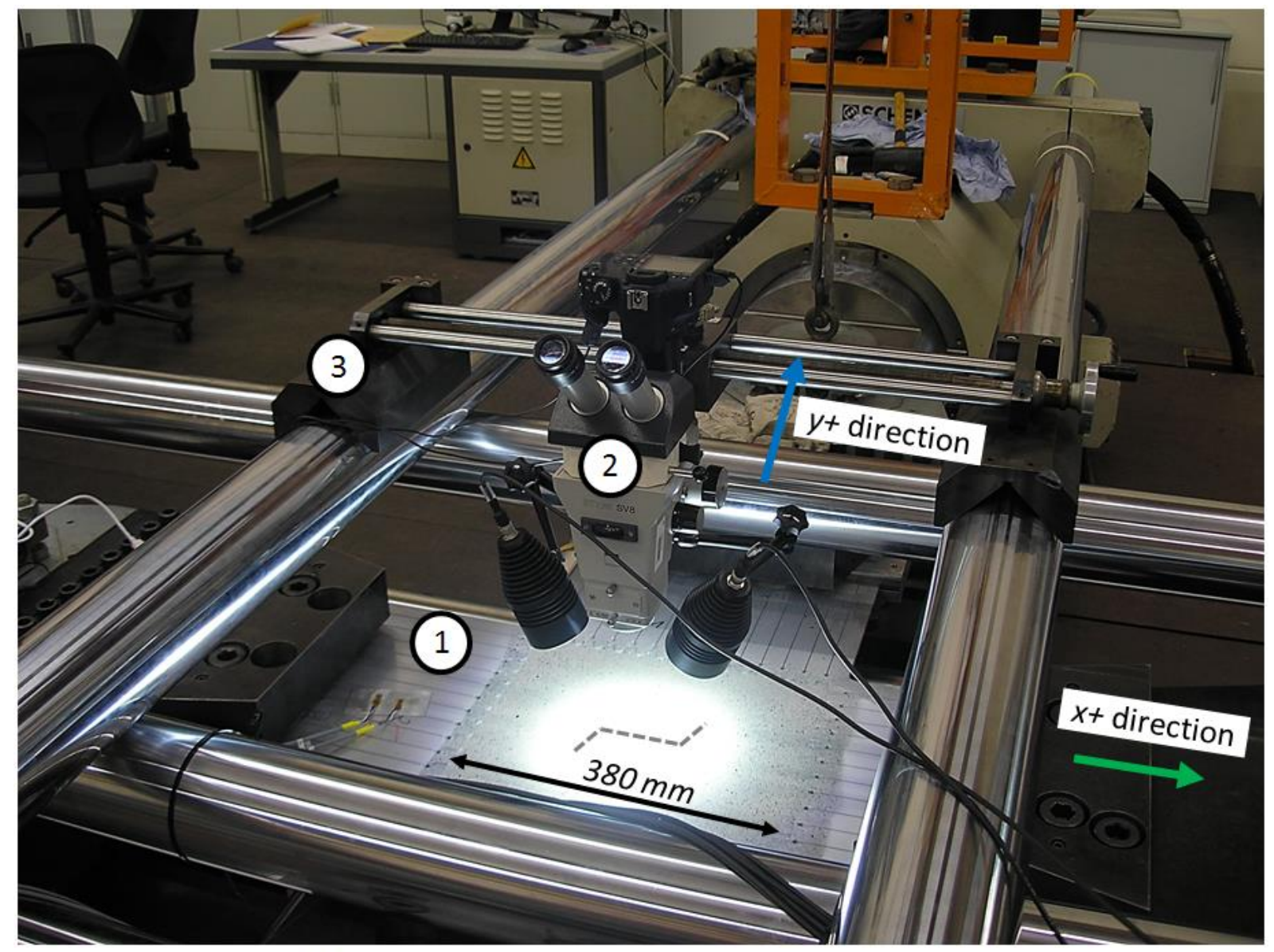

$\begin{array}{llll}\text { (1) Mounted specimen } & \text { (2) Microscope + DSLR } & \text { (3) Positioning device }\end{array}$

Figure 3: $\quad$ Experimental setup of the biaxial testing machine 
In the first part of the experiments the fatigue crack was loaded with $\lambda=0.0$, i.e. stress parallel to the crack was zero. This was done to obtain initially stable crack propagation perpendicular to the main loading axis (y-direction). Then the experiments with successively increasing biaxiality ratio were conducted as described above.

\section{Experimental results and discussion}

For a first estimation of the expected crack path under biaxial loadings FE simulations were conducted. Here the FE model of the biaxial specimen was used as described in the previous section. An artificial crack path was defined which is shown in Figure 4 (a). This middle crack had a total length $2 a=130 \mathrm{~mm}$ while the last $20 \mathrm{~mm}$ on each end were kinked by an angle between $0^{\circ}$ and $90^{\circ}$. Its basically (w.r.t. its center) centrosymmetric zig-zag shape was chosen based on the previous experimental observations. The model was loaded by $90 \mathrm{kN}$ in the $\mathrm{x}$ direction and $30 \mathrm{kN}$ in the $\mathrm{y}$ direction, i.e. $\lambda$ was 3.0. Based on the concept of stress intensity factors and the maximum tangential stress criterion [19] it is assumed that the crack path remains stable if $K_{\mathrm{II}}$ is zero. Basically, the same concept can be identified in Equation 2, as in linear-fracture mechanics it is generally assumed that a crack grows straight under pure Mode I conditions [20]. Figure 4 (b) shows that $K_{\mathrm{II}}=0 \mathrm{MPa} \sqrt{\mathrm{m} \text { occurs }}$ at kinking angles of $0 \mathrm{deg}$ and $75 \mathrm{deg}$ which means that a propagating fatigue crack loaded with $\lambda=3$ has basically two stable configurations. The crack path could remain straight or it could kink at 75 deg. The diagram also shows that for this configuration $K_{\mathrm{I}}$ increases with an increasing kink angle and reaches a maximum at approx. $80 \mathrm{deg}$, while $K_{\text {II }}$ has its minimum at about $40 \mathrm{deg}$. In this case higher absolute values of $K_{\text {II }}$ will have a higher tendency to cause kinking as compared to lower values. Furthermore it is shown that the $T$-stress is positive for kink angles lower than 13 deg which means that the crack path is basically prone to crack deflection as mentioned in the introduction.
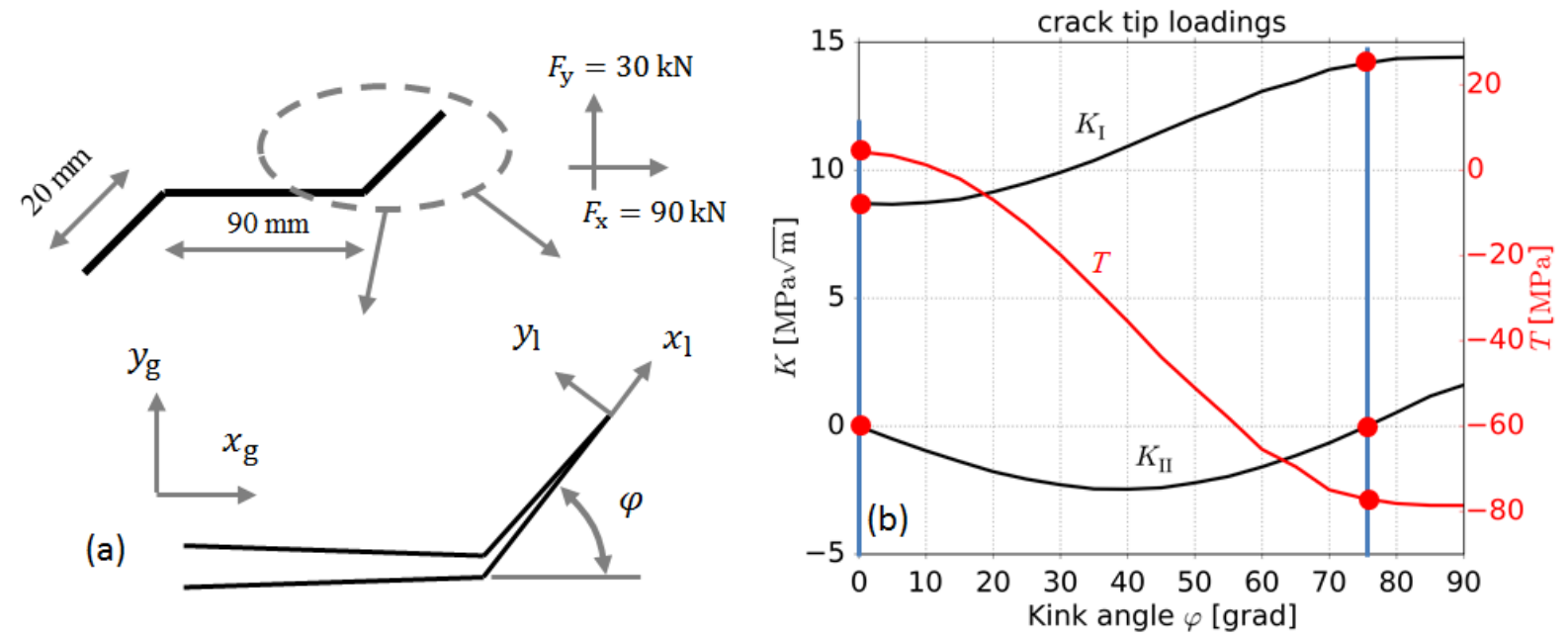

Figure 4: Artificial crack deflection in simulation. (a) Shape of the artificial crack path, (b) Results in terms of $K_{I}$, $K_{\text {II }}$ and $T$ dependent on the kink angle

In the next step the crack path was simulated for biaxiality ratios $\lambda$ between 0.0 and 3.0. The initial crack path was aligned in the $\mathrm{x}$-direction and had a length of $2 a=80 \mathrm{~mm}$. Without any geometrical disturbance at the crack tip the crack grew perfectly symmetrically independent of the biaxiality ratio or the $T$-stress. Under these conditions the crack tip has a pure mode I opening without any shear loadings. Therefore, in the following simulations the initial crack was given a small deviation by an orientation of $45^{\circ}$ and a length of $1.5 \mathrm{~mm}$ centrosymmetric at both ends. This representative flaw size is well in agreement with the chosen step size of $2 \mathrm{~mm}$ and with the mesh refinement used in our studies. In general much smaller geometric disturbances are also highly likely for real crack paths due to micro inhomogeneities or flaws present along the crack path [21]. Without a mode II loading it is 
not possible to predict the direction of the crack kinking (i.e. whether $+\varphi$ or $-\varphi$ ) because the sign of $K_{\text {II }}$ determines the direction of the kinking angle.

Figure 5 shows exemplarily the gauge area of the AA2024-T351 specimen with the kinked crack after the test has been finished. It can be seen in subfigure (a) that the crack grew almost straight after kinking. The equivalent von Mises strain in subfigure (b) reveals the strain redistribution after the crack kinked. While there are higher strains in the vicinity of the crack tip (green to red colors), the strain state near the crack faces (blue) in the upper center of the picture is significantly reduced as here the kinked crack provides a shielding effect against both loading axes.

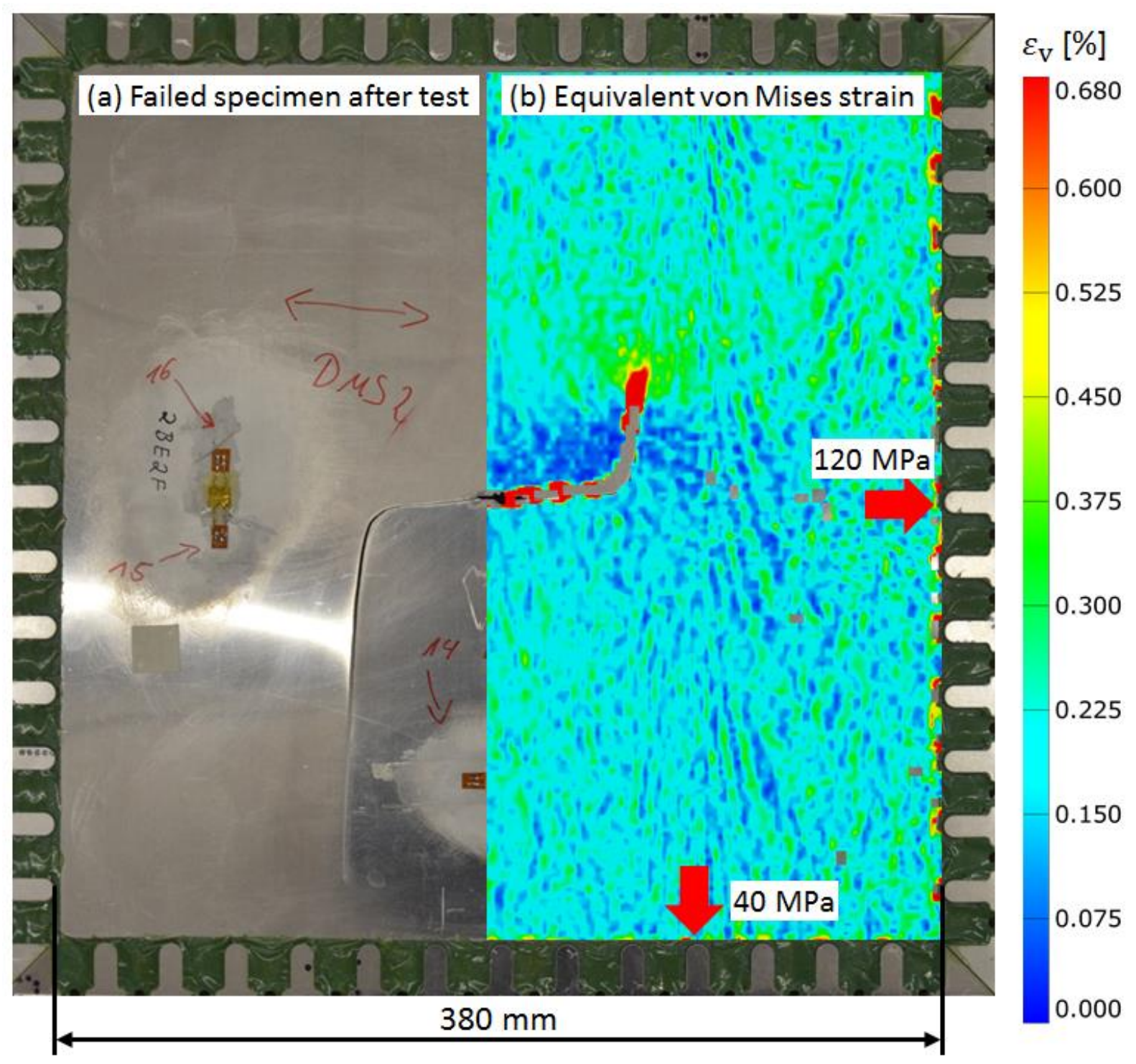

Figure 5: AA2024-T351 specimen after test with kinked crack (a) and DIC analysis showing equivalent von Mises strain at maximum loading conditions after crack kinking (b).

The results of the simulations and experiments are summarized in Figure 6. Even under a biaxiality ratio of 1.0 the crack grows almost straight. Significant deviations were noticed only for $\lambda>2.0$. At $\lambda=3.0$ the simulation predicts an angle of $\sim 85^{\circ}$ for the stable crack propagation direction which is in fairly good accordance to the simple model in Figure 4. 

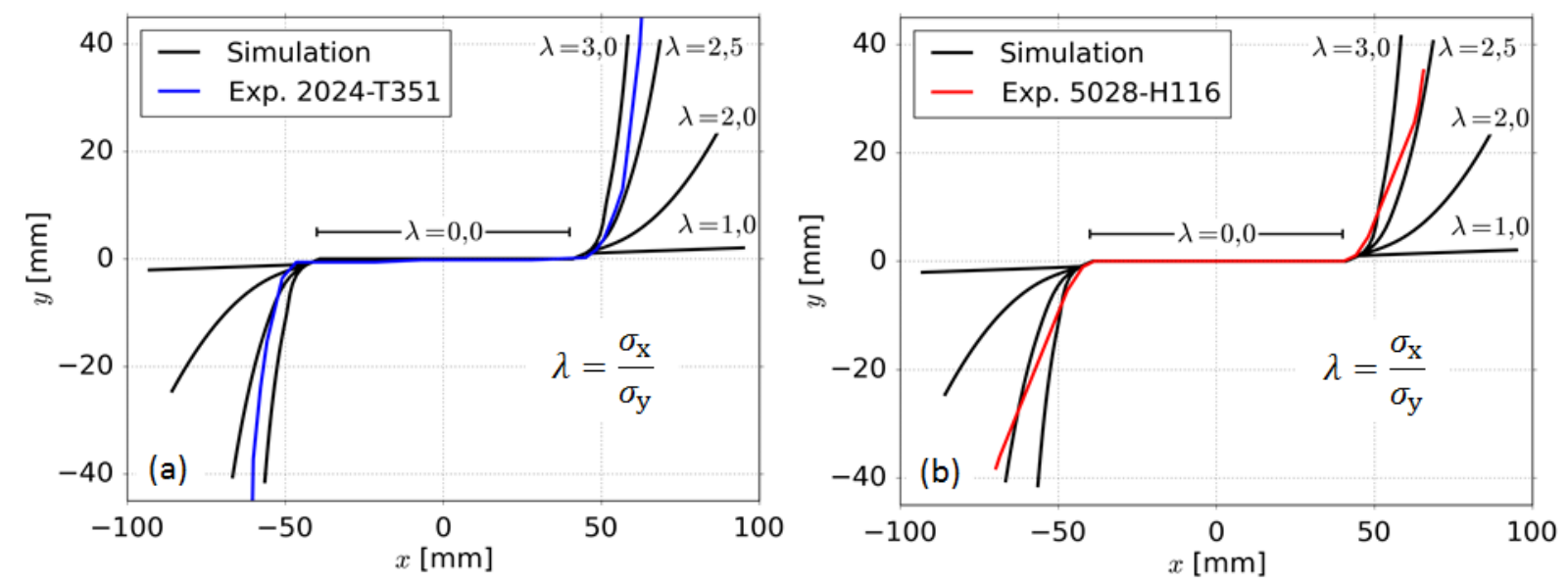

Figure 6: Simulated and experimental crack paths of biax tests. (a) Crack paths for the AA2024-T351 and (b) AA5028-H116 specimen. The experiments were conducted with $\lambda=3.0$

The experimental results $(\lambda=3.0)$ of the AA2024-T351 specimen are basically in good agreement with the simulations (see Figure 6 (a)). For the AA5028-H116 specimen the deviations are slightly higher, as the slope of the stable crack path is lower. Consequently, in AA5028-H116 the crack path seems to be less affected by biaxiality, i.e. it can "withstand" small $K_{\text {II }}$ loadings (see Figure 6 (b)) without immediately changing its main propagation direction. Consequently, the fatigue crack growth behavior in AA5028-H116 was investigated in more detail. Figure 7 shows the crack path after crack propagation under different biaxial fatigue loadings investigated by digital image correlation.

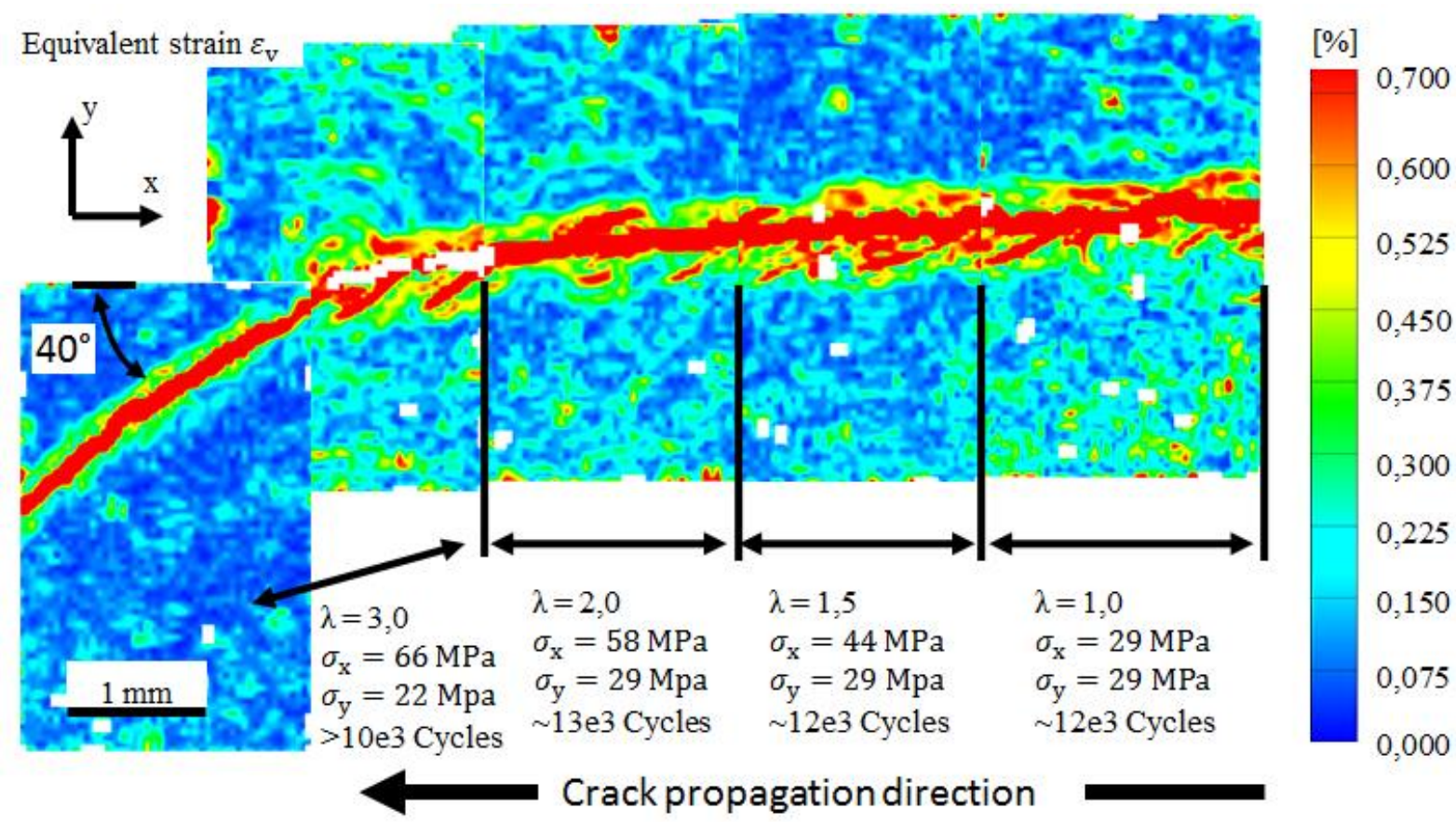

Figure 7: Local DIC analysis of the AA5028-H116 specimen's crack path at the loading phase with $\lambda>0$ and $\Delta K_{\mathrm{I}} \approx 10 \mathrm{MPa} \sqrt{\mathrm{m}}$. The contour plot shows the equivalent von Mises strain after unloading.

The pseudo-colors show the equivalent (von Mises) strain after unloading and therefore represent the plastic deformations and corresponding residual elastic strains. In this phase the crack was loaded with $\Delta K_{\mathrm{I}} \approx 6-10 \mathrm{MPa} \sqrt{\mathrm{m}}$ and the biaxiality ratio $\lambda$ was stepwise increased as indicted in the picture. Because of the artefacts by the DIC evaluations the crack path is indicated in red due to falsely computed high strains. First remarkable observation is that the crack does not kink for biaxial loads $\lambda \leq 2.0$, i.e. this behavior cannot accurately be predicted by the maximum tangential stress criterion (see crack path for $\lambda=2.0$ in Figure 6 (b)). In the phase before the crack actually kinks small "red 
spikes" are visible on both sides of the crack path. These spikes are either pronounced plastic deformation or even weakly pronounced cracks which cause the initial disturbances required for the actual crack deflection. This observation basically justifies the procedure of "artificial crack deflection" (see Figure 4) applied during the simulations. Furthermore, the red spikes in Figure 7 clearly indicate the direction of the upcoming crack kinking. Especially the two spikes just before kinking under $\lambda=3.0$ are parallel to the later crack path. After kinking the crack path does not show this kind of red spikes / disturbances anymore. This could be addresses to the significant reduction of the $T$-stress illustrated in Figure 4 (b). At a kinking angle of $\sim 40^{\circ}$ the $T$-stress has a value of about $-40 \mathrm{MPa}$. With this amount of negative $T$-stress the crack path remains basically stable and only slightly kinks during further crack propagation until $K_{\mathrm{II}}$ is almost zero. Although the underlying mechanisms behind these red spikes could not be further investigated within our current studies it can still be concluded, that they directly reflect the individual material's response to different biaxial loading conditions which also include the $T$-stress.

The local mechanical loadings acting for the AA2024-T351 and AA5028-H116 (compare Figure 7) specimens were analyzed in terms of $K_{\mathrm{I}}, T$-stress and nominal stresses, see Figure 8 . Here the FE model with a straight crack of a length of $2 a=90 \mathrm{~mm}$ was used and the nominal stresses were varied between 0 and $140 \mathrm{MPa}$ for $\sigma_{\mathrm{x}}$ and 0 and $50 \mathrm{MPa}$ for $\sigma_{\mathrm{y}}$. The color plot (Figure 8) represents the $T$ stress acting at the crack tip. All crack propagation phases for $\lambda<2.0$ have negative $T$-stresses. With the experiments it was shown that the crack paths remained straight as predicted based on literature [7], [9]. Then the positive $T$-stresses in the case of $\lambda=3.0$ lead immediately to an actually kinking crack. It has to be kept in mind the $T$-stress is generally not equal to the nominal stress in x-direction (parallel to the crack). Based on the diagram only for $\sigma_{\mathrm{y}}=0.0$ the $T$-stress is almost equal to the nominal stress. For constant $\sigma_{\mathrm{x}}$ the $T$-stress decreases with increasing $\sigma_{\mathrm{y}}$. It has to be emphasized that the $T$-stress is just an indicator if a crack deflection is generally possible. But if two potential crack paths are analyzed, the values of the $T$-stresses will clearly indicate which of these paths is more likely (see Figure 4 (b) again).

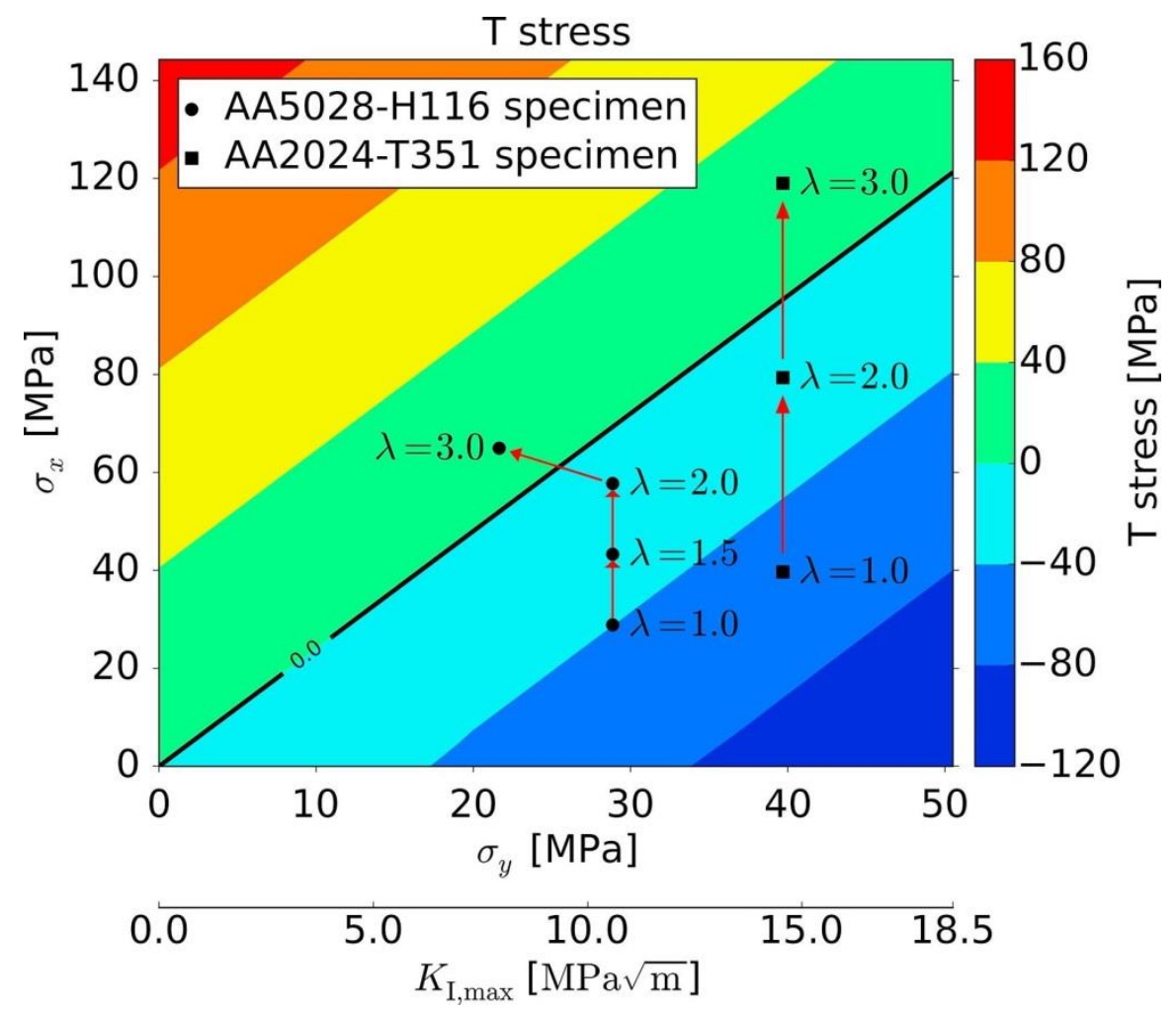

Figure 8: $T$-stress according to the nominal stresses of the $420 \times 420 \mathrm{~mm}^{2}$ specimens or $K_{\mathrm{I}}$ respectively. The crack is orientated parallel to the $x$ axis and had a length of $2 a=90 \mathrm{~mm}$. 


\section{Summary and Conclusions}

Biaxial tests for the aluminum alloys AA2024-T351 and AA5028-H116 were conducted to study the crack paths under biaxial fatigue loading conditions. Here the initial crack propagation was always parallel to one principal axis. The experiments were supported by a digital image correlation system to study the local materials response in terms of strain at local crack tip. For all experiments additional FE-simulations were conducted. Before drawing the final conclusions, it should be kept in mind that only two specimens were tested. But in both cases the crack propagation phases with increasing $\lambda$ consistently showed that the cracks turned / kinked immediately after the $T$-stresses reached positive values. Although this limited data base does not provide a reliable statistical validation the consistency of the findings clearly supports the following conclusions:

(1) For higher biaxiality ratios $(\lambda=3.0)$ the crack paths in both aluminium alloys can be sufficiently predicted by simulations using the well-known maximum tangential stress criterion. The difference between prediction and actual crack path is slightly higher for AA5028-H116.

(2) AA5028-H116: For lower biaxiality ratios $(\lambda \leq 2.0)$ the main fatigue crack does not change its direction, but pronounced plastic deformation / secondary cracks are observed along the crack path mainly tending towards the kinking direction observed for $\lambda=3.0$.

(3) AA5028-H116: This straight crack path under biaxial loadings up to $\lambda=2.0$ cannot accurately be predicted using the maximum tangential stress criterion.

(4) Analyses of $T$-stresses for AA2024-T351 and AA5028-H116 experiments confirmed that the criterion provided by Cotterell and Rice [7], [9] can be used to evaluate whether a crack will kink. For biaxiality ratios up to $\lambda=2.0$ all $T$-stresses were negative, while for $\lambda=3.0$ positive $T$-stresses were achieved causing kinking of the crack during further cycling. So positive $T$ stresses lead to crack kinking while at negative $T$-stresses the crack path remains stable.

(5) AA5028-H116: After kinking the new crack path did not show additional plastic deformations or secondary cracks as mentioned in conclusion (2). Consequently, the absence of these effects can be attributed to the presence of $T$-stresses $<<0$.

(6) As the $T$-stress based criterion does not yield the direction of crack kinking, additional analyses e.g. of maximum tangential stress are necessary to predict the crack path.

\section{Acknowledgement}

This work has been supported by the German Federal Ministry for Economic Affairs and Energy (BMWi) through the project MetLife embedded in the German aeronautic research fund LuFo 20142017 (code 20W1302B). The authors would like to thank J. Wischek, I. Druschke and C. Sick for conducting and supporting the experimental investigations.

\section{References}

[1] J. Schijve, "Fatigue damage in aircraft structures, not wanted, but tolerated?," International Journal of Fatigue, no. 31, p. 998-1011, 2009.

[2] L. Llopart, B. Kurz, C. Wellhausen, M. Anglada, K. Drechsler and K. Wolf, "Investigation of fatigue crack growth and crack turning on integral stiffened structures under mode I loading," Engineering Fracture Mechanics, no. 73, pp. 2139-2152, 2006.

[3] R. Pettit, J. C. Newmann and M. S. Domack, "Crack turning damage tolerance approach for integrally stiffened structure," in 19th ICAF symposium, Edinburgh, 1997. 
[4] R. G. Pettit, Crack turning in integrally stiffened aircraft structures, Cornell University, 2000.

[5] M. L. Williams, "On the Stress Distribution at the Base of a Stationary Crack," Journal of Applied Mechanics, no. 24, pp. 109-114, 1956.

[6] G. R. Irwin, "Analysis of stresses and strains near the end of a crack traversing a plate," Journal of Applied Mechanics, no. 24, pp. 361-364, 1957.

[7] B. Cotterell, "Notes on the paths and stability of cracks," International Journal of Fracture Mechanics, no. 2, pp. 526-533, 1966.

[8] C. She and W. Guo, "The out-of-plane constraint of mixed-mode cracks in thin elastic plates," International Journal of Solids and Structures, no. 44, pp. 3021-3034, 2007.

[9] B. Cotterell and J. Rice, "Slightly curved or kinked cracks," International Journal of Fracture, vol. 2, no. 12, pp. 155-169, 1980.

[10] C.-S. Chen, P. A. Wawrzynek and A. R. Ingraffea, "Prediction of Residual Strength and Curvilinear Crack Growth in Aircraft Fuselages," AIAA JOURNAL, vol. 8, no. 40, pp. 1644-1652, 2002.

[11] Y. G. Matvienko, "MAXIMUM AVERAGE TANGENTIAL STRESS CRITERION FORPREDICTION OF THE CRACK PATH," International Journal of Fracture, no. 176, pp. 113-118, 2012.

[12] J. Sobotka and R. Dodds Jr., "T-stress effects on steady crack growth in a thin, ductile plate under small-scale yielding conditions: Three-dimensional modeling," Engineering Fracture Mechanics, no. 78, pp. 1182-1200, 2011.

[13] M. Besel and E. Breitbarth, "Advanced analysis of crack tip plastic zone under cyclic loading," International Journal of Fatigue, no. 93, p. 92-108, 2016.

[14] E. Breitbarth and M. Besel, "Energy based analysis of crack tip plastic zone of AA2024-T3 under cyclic loading," International Journal of Fatigue, no. 100, pp. 263-273, 2017.

[15] E. Breitbarth, M. Besel and S. Reh, "Biaxial testing of cruciform specimens representing characteristics of a metallic airplane fuselage section," International Journal of Fatigue, no. 108, pp. 116-126, 2018.

[16] Department of Defense, MIL-HDBK-5J - Metallic Materials and Elements for Aerospace Vehicle Structures, 2003.

[17] H. A. Richard, F. G. Buchholz, G. Kullmer and M. Schöllmann, "2D- and 3D-Mixed Mode Fracture Criteria," Engineering Materials, no. 251, pp. 251-260, 2003.

[18] "GOM GmbH," 2017. [Online]. Available: http://www.gom.com/.

[19] F. Erdogan and C. Sih, "On the Crack Extension in Plates Under Plane Loading and Transverse Shear," Journal of Basic Engineering, no. 85, pp. 519-525, 1963.

[20] M. Kuna, Finite Elements in Fracture Mechanics, Dordrecht: Springer Science+Business Media, 2013.

[21] D. Leguillon and S. Murer, "Crack deflection in a biaxial stress state," International Journal of 
Fracture, no. 150, p. 75-90, 2008. 